\title{
NOUVELLE CUISINE FOR THE COMPUTATION OF THE ANNIHILATING IDEAL OF $f^{s}$
}

\author{
J. GAGO-VARGAS, M.I. HARTILLO-HERMOSO, AND J.M. UCHA-ENRÍQUEZ
}

\begin{abstract}
Let $f_{1}, \ldots, f_{p}$ be polynomials in $\mathbf{C}\left[x_{1}, \ldots, x_{n}\right]$ and let $D=D_{n}$ be the $n$-th Weyl algebra. The annihilating ideal of $f^{s}=f_{1}^{s_{1}} \ldots f_{p}^{s_{p}}$ in $D[s]=$ $D\left[s_{1}, \ldots, s_{p}\right]$ is a necessary step for the computation of the Bernstein-Sato ideals of $f_{1}, \ldots, f_{p}$.

We point out experimental differences among the efficiency of the available methods to obtain this annihilating ideal and provide some upper bounds for the complexity of its computation.
\end{abstract}

\section{INTRODUCTION}

Fix two integers $n \geq 1, p \geq 1$ and two sets of variables $\left(x_{1}, \ldots, x_{n}\right)$ and $\left(s_{1}, \ldots, s_{p}\right)$. Let us consider $f_{1}, \ldots, f_{p} \in \mathbf{C}[x]=\mathbf{C}\left[x_{1}, \ldots, x_{n}\right]$ and let $D=D_{n}$ be the $n$-th Weyl algebra. A polynomial $b(s) \in \mathbf{C}[s]=\mathbf{C}\left[s_{1}, \ldots, s_{p}\right]$ is said to be a Bernstein-Sato polynomial associated to $f$ if the following functional equation holds for a certain $P(s) \in D[s]:$

$$
b(s) f^{s}=P(s) f^{s+1},
$$

where $\mathbf{1}=(1, \ldots, 1)$. These polynomials form an ideal called the Bernstein-Sato ideal $\mathcal{B}_{f}$, or simply $\mathcal{B}$ to abbreviate. Analogous functional equations with respect to vectors different to $\mathbf{1}$ yield other different Bernstein-Sato ideals (see for example $[\mathrm{Ba} 1])$.

In [L1] it is proved that $\mathcal{B}$ is not zero. This fact is a generalization of the classical proof of Bernstein ([Be1]) for the case $p=1$, in which the generator of $\mathcal{B}$ is called the Bernstein-Sato polynomial, $b_{f}(s)$. The analytical work was made in $[\mathrm{Bj} 1]$ for $p=1$ and in [Sa1],[Sa2] for $p>1$.

The roots of $b_{f}(s)$ encode important algebro-geometrical data (see [Mal1], [H1] or [BS1] to mention only a few) and a complete understanding of all roots for a general $f$ is open. For the case $p>1$ there is a lot of work to do yet: there are conjectures on the primary decomposition of $\mathcal{B}$, on the conditions over $f$ for $\mathcal{B}$ to be principal, to be zero-dimensional, etc.

In [O1] was presented the first algorithm to find the Bernstein-Sato polynomial, and alternative methods have been proposed to obtain $\mathcal{B}$ in the general case in [OT1], [Ba1] and [BM1]. All these methods have a feature in common: their first step is the computation of the annihilating ideal of $f^{s}$ in $D[s], A n n_{D[s]} f^{s}$. We recall here some experimental evidences in favor of the method of Briançon-Maisonobe [BM1] with respect to the method of Oaku-Takayama [OT1].

Then we will give upper bounds of the complexity of computing $A n n_{D[s]} f^{s}$, the previous requirement for both algorithms. To obtain this bounds we use - as far as possible - the techniques and results of [Gr1] on the complexity of solving systems

All authors partially supported by MTM2004-01165 and FQM-333. 
of linear equations over rings of differential operators (that extend the classical polynomial case treated in [Se1]). In particular, we show that the construction of Grigoriev can not be directly generalized to any non-commutative algebra, including the algebra proposed by Briançon-Maisonobe. We prove that the complexity of computing $A n n_{D[s]} f^{s}$ using the method of [BM1] is that of the calculation of a Gröbner basis in the $n$-th Weyl algebra with some extra $p$ commutative variables $(2 n+p$ variables at most), while in the case of the method [OT1] is the calculation of such a basis in a $(n+p)$-th Weyl algebra with some extra $2 p$ variables (so $2 n+4 p$ variables in all).

We are very grateful to the referees for helping us to clarify our initial version.

\section{Preliminaries}

In this section we just remind briefly some details of the methods of BriançonMaisonobe and Oaku-Takayama, respectively.

2.1. Method of Briançon-Maisonobe. In this case the computations are made in the non-commutative algebra

$$
R=D_{n}[s, t]=D_{n}\left[s_{1}, \ldots, s_{p}, t_{1}, \ldots, t_{p}\right],
$$

an extension of the $n$-th Weyl algebra $D$ in which the new variables $s, t$ satisfy the relations $\left[s_{i}, t_{j}\right]=\delta_{i j} t_{i}$. It is a a Poincaré-Birkhoff-Witt $(P B W)$ algebra:

Definition 1. A PBW algebra $R$ over a ring $k$ is an associative algebra generated by finitely many elements $x_{1}, \ldots, x_{n}$ subject to the relations

$$
Q=\left\{x_{j} x_{i}=q_{j i} x_{i} x_{j}+p_{j i}, 1 \leq i<j \leq n\right\},
$$

where each $p_{j i}$ is a finite $k$-linear combination of standard terms $\mathbf{x}^{\alpha}=x_{1}^{\alpha_{1}} \cdots x_{n}^{\alpha_{n}}$ and each $q_{j i} \in k$ with the two following conditions:

(1) There is an admissible $e^{1}$ order $\prec$ on $\mathbf{N}^{n}$ such that $\exp \left(p_{j i}\right) \prec \exp \left(x_{j} x_{i}\right)$ for every $1 \leq i<j \leq n$.

(2) The standard terms $\mathbf{x}^{\alpha}$, with $\alpha \in \mathbf{N}^{n}$, form a $k$-basis of $R$ as a vector space.

It is possible to compute Gröbner bases in PBW algebras. The book [BGV1] is a good introduction to the subject of effective calculus in this fairly general family.

The following algorithm computes $\mathcal{B}$, starting from

$$
I:=A n n_{R}\left(f^{s}\right)=\left\langle s_{j}+f_{j} t_{j}, \partial_{i}+\sum_{j} \frac{\partial f_{j}}{\partial x_{i}} t_{j}, 1 \leq i \leq n, 1 \leq j \leq p\right\rangle .
$$

Algorithm 2.1. You have to:

(1) Obtain $J=$ Ann $_{D_{n}[s]} f^{s}=\left\langle G_{1} \cap D_{n}[s]\right\rangle$ where $G_{1}$ is a Gröbner basis of $I$ with respect to any term ordering with variables $t_{j}$ greater than the others (that is, an elimination ordering for the $t_{j}$.)

(2) $\mathcal{B}=\left\langle G_{2} \cap \mathbf{C}[s]\right\rangle$, where $G_{2}$ is a Gröbner basis of $J+\left(f_{1}, \ldots, f_{p}\right)$ with respect to any term ordering with $x_{i}, \partial_{j}$ greater than the $s_{l}$.

\footnotetext{
${ }^{1}$ Here admissible means a total order among the elements of $\mathbf{N}^{n}$ with $\mathbf{0}$ as least element.
} 
2.2. Method of Oaku-Takayama. All the computations are made in Weyl algebras. More precisely, starting from

$$
I^{\prime}=\left\langle t_{j}-f_{j}, \sum_{j=1}^{p} \frac{\partial f_{j}}{\partial x_{i}} \partial_{t_{j}}+\partial_{i}, i=1, \ldots, n, j=1, \ldots, p\right\rangle
$$

Algorithm 2.2. You have to:

(1) Obtain $J^{\prime}=I^{\prime} \cap \mathbf{C}\left[t_{1} \partial_{t_{1}}, \ldots, t_{n} \partial_{t_{n}}\right]\left\langle x, \partial_{x}\right\rangle$.

(2) $J=A n n_{D_{n}[s]}\left(f^{s}\right)=J^{\prime \prime}$, where $J^{\prime \prime}$ denotes the ideal generated by the generators of $J^{\prime}$ after replacing each $t_{i} \partial_{t_{i}}$ by $-s_{i}-1$.

(3) $\mathcal{B}=\left\langle G_{2} \cap \mathbf{C}[s]\right\rangle$, where $G_{2}$ is a Gröbner basis of $J+\left(f_{1}, \ldots, f_{p}\right)$ with respect to any term ordering with $x_{i}, \partial_{j}$ greater than the $s_{l}$.

The second step above ${ }^{2}$ is again the elimination of all the variables but $\left(s_{1}, \ldots, s_{p}\right)$. The computation of

$$
I^{\prime} \cap \mathbf{C}\left[t_{1} \partial_{t_{1}}, \ldots, t_{n} \partial_{t_{n}}\right]\left\langle x, \partial_{x}\right\rangle
$$

uses $2 n+4 p$ variables, as new variables $u_{j}, v_{j}$ for $1 \leq j \leq p$ are introduced. More precisely, the first calculation is an elimination of these new variables for the ideal

$$
\left\langle t_{j}-u_{j} f_{j}, \sum_{j=1}^{p} \frac{\partial f_{j}}{\partial x_{i}} u_{j} \partial_{t_{j}}+\partial_{i}, 1-u_{j} v_{j}, \quad 1 \leq i \leq n, 1 \leq j \leq p,\right\rangle,
$$

and some more technical steps must be followed (see [OT1, Procedure 4.1.]).

\section{EXPERIMENTAL DATA}

Here we give some examples for the cases $p=1,2$ and $p>2$ for which it is clear the superiority of Briançon-Maisonobe's method. They have been tested ${ }^{3}$ using Singular::Plural 2.1 (see [GLS1]) in a PC Pentium IV, 1Gb RAM and 3.06GHz running under Windows XP.

SingUlAR::PluRAL 2.1 is a system for non-commutative general purpose, so the calculations in our algebras are not supposed to be optimal. We present the following data only for the sake of comparing both methods in the same system. In the case of [BM1] method we have used a pure lexicographical ordering, while for [OT1] we have used typical elimination ordering. These are the orderings with best results for each case.

The typical input for Singular::PluRal 2.1 looks like this for [BM1] method: ring $r=0,(t(1 \ldots 3), s(1 \ldots 3), x, y, z, D x, D y, D z), l p$;

matrix $C[12][12]=0$;

$C[1,4]=t(1) ; C[2,5]=t(2) ; C[3,6]=t(3) ; C[7,10]=1 ; C[8,11]=1 ; C[9,12]=1 ;$

system("PLURAL" , 1,C);

poly $f 1=x * z+y ;$ poly $f 2=x * y+z ;$ poly $f 3=y * z+x$

\footnotetext{
${ }^{2}$ Often the bottleneck to obtain the Bernstein-Sato ideal is this step. As far as we know, the example for $p=2$ with $f_{1}=x^{2}+y^{3}, f_{2}=x^{3}+y^{2}$ is intractable for the available systems.

${ }^{3}$ The CPU times must be considered as approximations: as it is explained in the SinguLAR::PLURAL 2.1 Manual, the command timer is not absolutely reliable due to the shortcomings of the Windows operating system.
} 
TABLE 1. CPU times for the computation of Annf $f^{s}$

\begin{tabular}{|c||c|c|}
\hline$f$ & Briançon-Maisonobe's method & Oaku-Takayama's method \\
\hline$x^{3}+x y^{2}+z^{2}$ & $<0.01 \mathrm{~s}$ & $0.39 \mathrm{~s}$ \\
\hline$x^{4}+y^{3}+z^{2}$ & $<0.01 \mathrm{~s}$ & $0.39 \mathrm{~s}$ \\
\hline$y x^{3}+y^{3}+z^{2}$ & $0.06 \mathrm{~s}$ & $3.97 \mathrm{~s}$ \\
\hline \hline$x^{3}+y^{2}+z^{2}$ & $<0.01 \mathrm{~s}$ & $0.02 \mathrm{~s}$ \\
\hline$x^{5}+y^{2}+z^{2}$ & $<0.01 \mathrm{~s}$ & $4.66 \mathrm{~s}$ \\
\hline$x^{7}+y^{2}+z^{2}$ & $<0.01 \mathrm{~s}$ & $298.56 \mathrm{~s}$ \\
\hline \hline$x^{4}+y^{5}+x y^{4}$ & $0.56 \mathrm{~s}$ & $\mathrm{E}(>12 \mathrm{~h})$ \\
\hline
\end{tabular}

TABLE 2. CPU times for the computation of $A n n f_{1}^{s_{1}} f_{2}^{s_{2}}$

\begin{tabular}{|c|c||c|c|}
\hline$f_{1}$ & $f_{2}$ & Briançon-Maisonobe's method & Oaku-Takayama's method \\
\hline$x^{3}+y^{2}$ & $x^{2}+y^{3}$ & $0.72 \mathrm{~s}$ & $6363.97 \mathrm{~s}$ \\
\hline$x^{5}+y^{3}$ & $x^{3}+y^{5}$ & $3.53 \mathrm{~s}$ & $\mathrm{E}(>6 \mathrm{~h})$ \\
\hline$x^{7}+y^{5}$ & $x^{5}+y^{7}$ & $11.84 \mathrm{~s}$ & $\mathrm{E}(>6 \mathrm{~h})$ \\
\hline \hline$x^{3}+y^{2}$ & $x z+y$ & $<0.01 \mathrm{~s}$ & $9.73 \mathrm{~s}$ \\
\hline$x^{5}+y^{2}$ & $x z+y$ & $<0.01 \mathrm{~s}$ & $1568.59 \mathrm{~s}$ \\
\hline$x^{11}+y^{5}$ & $x z+y$ & $3 \mathrm{~s}$ & $\mathrm{E}(>6 \mathrm{~h})$ \\
\hline
\end{tabular}

ideal $i=s(1)+t(1) * f 1, s(2)+t(2) * f 2, s(3)+t(3) * f 3, D x+$ $t(1) * \operatorname{diff}(\mathrm{f} 1, \mathrm{x})+\mathrm{t}(2) * \operatorname{diff}(\mathrm{f} 2, \mathrm{x})+\mathrm{t}(3) * \operatorname{diff}(\mathrm{f} 3, \mathrm{x})$, Dy + $t(1) * \operatorname{diff}(f 1, y)+t(2) * \operatorname{diff}(f 2, y)+t(3) * \operatorname{diff}(f 3, y), D z+$ $t(1) * \operatorname{diff}(f 1, z)+t(2) * \operatorname{diff}(f 2, z)+t(3) * \operatorname{diff}(f 3, z)$;

ideal $I=\operatorname{std}(i)$;

And this one for [OT1] method:

ring $r=0,(u, v, x, y, z, t, D x, D y, D z, D t),(a(1,1), d p)$;

matrix $C[10][10]=0$;

$C[3,7]=1 ; C[4,8]=1 ; C[5,9]=1 ; C[6,10]=1 ;$

system("PLURAL", 1, C);

(1) Case $p=1$ : In the following examples $f \in \mathbf{C}[x, y]$ or $f \in \mathbf{C}[x, y, z]$. They have been chosen taking into account Arnold's classification of singularities. E means the memory was exhausted and the system reported an error.

(2) Case $p=2$ : In Table 2 the examples $f_{1}, f_{2}$ are in $\mathbf{C}[x, y]$ or $\mathbf{C}[x, y, z]$.

(3) Case $p>2$ : In Table 3 we have some examples for more than two functions. When the single functions $f_{1}, \ldots, f_{p}$ are "simple" enough (for example, linear) it is possible to obtain $A n n_{D\left[s_{1}, \ldots, s_{p}\right]} f_{1}^{s_{1}} \cdots f_{p}^{s_{p}}$ for $p$ rather big (say 15 or 20 ). This ideal can be related to the annihilating ideal of $f=f_{1} \cdots f_{p}$. 
TABLE 3. CPU times for the computation of $A n n f_{1}^{s_{1}} \cdots f_{p}^{s_{p}}$

\begin{tabular}{|c|c|c||c|c|}
\hline$f_{1}$ & $f_{2}$ & $f_{3}$ & Briançon-Maisonobe's method & Oaku-Takayama's method \\
\hline$x+y$ & $x-y$ & $x^{2}+y$ & $<0.01 s$ & $29.46 \mathrm{~s}$ \\
\hline$x+y$ & $x^{2}+y$ & $x+y^{2}$ & $2.64 \mathrm{~s}$ & $\mathrm{E}$ \\
\hline$x+y$ & $x^{2}+y$ & $x^{2}+y^{3}$ & $116.24 \mathrm{~s}$ & $\mathrm{E}$ \\
\hline$x+y$ & $x^{2}+y$ & $x^{3}+y^{2}$ & $1728.41 \mathrm{~s}$ & $\mathrm{E}$ \\
\hline
\end{tabular}

TABLE 4. Some arrangements of hyperplanes.

\begin{tabular}{|c||c|c|}
\hline$f=f_{1} \cdots f_{p}$ & $\begin{array}{c}\text { Briançon-Maisonobe's method } \\
\text { computing } \text { Ann }_{D\left[s_{1}, \ldots, s_{p}\right]} f_{1}^{s_{1}} \cdots f_{p}^{s_{p}}\end{array}$ & $\begin{array}{c}\text { Asir } \\
\text { computing } A n n_{D[s]} f^{s}\end{array}$ \\
\hline$x y z(x+y)(x-y)(x-2 y-z)$ & $0.62 \mathrm{~s}$ & $0.93 \mathrm{~s}$ \\
\hline$x y z(x-y)(x+y)(x-2 y)$ & $0.05 \mathrm{~s}$ & $0.03 \mathrm{~s}$ \\
\hline$x y z(x+y)(x-y)(x+y-z)$ & $0.06 \mathrm{~s}$ & $3.54 \mathrm{~s}$ \\
\hline$x y z u(x+y+z+u)$ & $0.01 \mathrm{~s}$ & $6.99 \mathrm{~s}$ \\
\hline$x y z u v w(x+y+z+u+v)$ & $0.02 \mathrm{~s}$ & $1691.31 \mathrm{~s}$ \\
\hline
\end{tabular}

This idea has been exploited with success in [GHU1] to compute annihilating ideals for $f$, where $f$ defines very hard examples of arrangements of hyperplanes of theoretical interest. In Table 4 we compare the results of applying this idea in Singular::Plural 2.1 with obtaining directly $A n n_{D[s]} f^{s}$ using the powerful system Asir (see [N1]).

\section{Complexity}

In [Gr1] a bound for the degree of the solutions of a general system of linear equations over the Weyl algebra is given, with a procedure somewhat similar to the one of the commutative case of [Se1]. In this section we study how far the work of Grigoriev is applicable to our PBW algebra $R$ of 2.1. His construction has two different steps: in the first, the given system is reduced to another system in a diagonal form. In the second, it is shown how to normalize the new system in order to eliminate, successively, the variables.

4.1. Diagonalization. We need three technicals lemma to reduce the system to a diagonal form. They generalize analogous lemmas of Grigoriev's paper (see [Gr1, Lemma 1]) and their proofs are, more or less, straightforward. Here deg means the total degree of a term, that is, the sum of the exponents of all its variables.

Lemma 1. Let $A$ be a $(m-1) \times m$ matrix with entries in a Poincaré-Birkhoff-Witt algebra $S$ with a basis of $p$ elements. If $\operatorname{deg}\left(a_{i j}\right) \leq d$, there exists a nonzero-vector $f=\left(f_{1}, \ldots, f_{m}\right) \in S^{m}$ such that $A f=0$ and $\operatorname{deg}(f) \leq 2 p(m-1) d=N$.

If we work in a noetherian domain (eventually non-commutative), we can always define the rank of a finite module as in [St1]. Given a square matrix in a PoincaréBirkhoff-Witt algebra we say that it is non-singular if it has maximal rank, and in this case we can obtain a left quasi-inverse with the precedent lemma: 
Lemma 2. Given a $m \times m$ matrix $B$ over a PBW algebra $S$ as in Lemma 1, non-singular, it has a left quasi-inverse matrix $G$ over $S$, such that $\operatorname{deg}(G) \leq N$.

Lemma 3. Given a system of linear equations over a $P B W$ algebra $S$, it is defined by a $m \times s$ matrix $A$ of rank $r$, with its elements $\operatorname{deg}\left(a_{i j}\right) \leq d$ we can always construct a matrix $C$, which defines an equivalent system, such that

$$
C A=\left(\begin{array}{cc}
C_{1} & 0 \\
C_{2} & E
\end{array}\right) A=\left(\begin{array}{ccc|c}
a_{1} & & 0 & \\
& \ddots & & \star \\
0 & & a_{r} & \\
\hline & 0 & & 0
\end{array}\right)
$$

where $E$ is the identity matrix.

Due to this lemma, we can assume that our system is equivalent to a system in diagonal form:

$$
a_{k} V_{k}+\sum_{r+1 \leq l \leq s} a_{k, l} V_{l}=b_{k}, \quad 1 \leq k \leq r, \quad \operatorname{deg}\left(a_{k}\right), \operatorname{deg}\left(a_{k, l}\right), \operatorname{deg}\left(b_{k}\right) \leq 2 p m d .
$$

4.2. Normalization. Once the system is in diagonal form, we need to normalize it. To do this, we construct some syzygies, applying Lemma 1 to the submatrix of the first $r$ columns and the column $l>r$. There always exist $h^{(l)}, h_{1}^{(l)}, \ldots, h_{r}^{(l)}$ such that:

$$
a_{k} h_{k}^{(l)}+a_{k, l} h^{(l)}=0, \quad 1 \leq k \leq r \quad \operatorname{deg}\left(h^{(l)}\right), \operatorname{deg}\left(h_{i}^{(l)}\right) \leq 4 p^{2} m^{2} d
$$

The result that gives the normalization in the Weyl algebra is the following one:

Lemma 4 ([Gr1], Lemma 4). Given $g_{1}, \ldots, g_{t} \in D_{n}$ a family of elements, there is a nonsingular linear transformation of $2 n$-dimensional space with basis $x_{1}, \ldots, x_{n}, \partial_{1}, \ldots, \partial_{n}$ under which:

$$
\begin{aligned}
x_{i} \rightarrow \Gamma_{x_{i}} & =\sum_{j=1}^{n} \gamma_{i, j}^{(1,1)} x_{j}+\sum_{j=1}^{n} \gamma_{i, j}^{(1,2)} \partial_{j} ; \\
\partial_{i} \rightarrow \Gamma_{\partial_{i}} & =\sum_{j=1}^{n} \gamma_{i, j}^{(2,1)} x_{j}+\sum_{j=1}^{n} \gamma_{i, j}^{(2,2)} \partial_{j}
\end{aligned}
$$

such that the following relations hold:

$$
\begin{gathered}
\Gamma_{x_{i}} \Gamma_{\partial_{i}}=\Gamma_{\partial_{i}} \Gamma_{x_{i}}-1 ; \quad \Gamma_{x_{i}} \Gamma_{x_{j}}=\Gamma_{x_{j}} \Gamma_{x_{i}} \\
\Gamma_{\partial_{i}} \Gamma_{\partial_{j}}=\Gamma_{\partial_{j}} \Gamma_{\partial_{i}} \quad \Gamma_{\partial_{i}} \Gamma_{x_{j}}=\Gamma_{x_{j}} \Gamma_{\partial_{i}} \quad i \neq j,
\end{gathered}
$$

and if we denote by $\Gamma_{g_{i}}$ the transformed of $g_{i}$ with the indicated linear transformation, we have $\Gamma_{g_{i}}=\partial_{n}^{\operatorname{deg}\left(g_{i}\right)}+\widetilde{\Gamma_{g_{i}}}$.

Remark 1. The main fact in the proof of the last Lemma 4 is that the matrices of the linear transformations defined by the relations in the Weyl algebra are a transitive group.

Let $\left\{g_{1}, \ldots, g_{t}\right\}$ be a set of elements in $R=\mathbf{C}\left[s, t, x_{1}, \ldots, x_{n}, \partial_{1}, \ldots, \partial_{n}\right]$. Let us see why we can not assure the existence of a linear transformation $\Gamma$ that produces

$$
\Gamma_{g_{i}}=v^{\operatorname{deg}\left(g_{i}\right)}+\widetilde{\Gamma_{g_{i}}},
$$

where $v$ is a single variable.

A general linear transformation as the one postulated in Lemma 4 has the form: 


$$
\begin{aligned}
& s \rightarrow \Gamma_{s}=\alpha_{1} s+\beta_{1} t \quad+\sum_{j=1}^{n} \gamma_{j}^{(s, 1)} x_{j}+\sum_{j=1}^{n} \gamma_{j}^{(s, 2)} \partial_{j} \\
& t \rightarrow \Gamma_{t}=\alpha_{2} s+\beta_{2} t+\sum_{j=1}^{n} \gamma_{j}^{(t, 1)} x_{j}+\sum_{j=1}^{n} \gamma_{j}^{(t, 2)} \partial_{j} \\
& x_{i} \rightarrow \Gamma_{x_{i}}=\alpha_{i}^{(1)} s+\beta_{i}^{(1)} t+\sum_{j=1}^{n} \gamma_{i, j}^{(1,1)} x_{j}+\sum_{j=1}^{n} \gamma_{i, j}^{(1,2)} \partial_{j} \\
& \partial_{i} \rightarrow \Gamma_{\partial_{i}}=\alpha_{i}^{(2)} s+\beta_{i}^{(2)} t+\sum_{j=1}^{n} \gamma_{i, j}^{(2,1)} x_{j}+\sum_{j=1}^{n} \gamma_{i, j}^{(2,2)} \partial_{j}
\end{aligned}
$$

and it has to verify the following relations:
(1) $\Gamma_{s} \Gamma_{t}=\Gamma_{t} \Gamma_{s}+\Gamma_{t}$
(2) $\Gamma_{s} \Gamma_{x_{i}}=\Gamma_{x_{i}} \Gamma_{s}$
(3) $\Gamma_{s} \Gamma_{\partial_{i}}=\Gamma_{\partial_{i}} \Gamma_{s}$;
(4) $\Gamma_{t} \Gamma_{x_{i}}=\Gamma_{x_{i}} \Gamma_{t}$
(5) $\Gamma_{t} \Gamma_{\partial_{i}}=\Gamma_{\partial_{i}} \Gamma_{t}$
(6) $\Gamma_{x_{i}} \Gamma_{\partial_{i}}=\Gamma_{\partial_{i}} \Gamma_{x_{i}}-1$;
(7) $\Gamma_{x_{i}} \Gamma_{x_{j}}=\Gamma_{x_{j}} \Gamma_{x_{i}}$;
(8) $\Gamma_{\partial_{i}} \Gamma_{\partial_{j}}=\Gamma_{\partial_{j}} \Gamma_{\partial_{i}}$
(9) $\Gamma_{x_{i}} \Gamma_{\partial_{j}}=\Gamma_{\partial_{j}} \Gamma_{x_{i}}$

From relation (1), we obtain $\alpha_{2}=\gamma_{j}^{(t, 1)}=\gamma_{j}^{(t, 2)}=0$ for all $j$, so $\Gamma_{t}=\beta_{2} t$. The change must be nonsingular, so we have $\beta_{2} \neq 0$, and again using relation (1) we deduce that $\alpha_{1}=1$. Using relation (4), we obtain that $\alpha_{i}^{(1)}=0$ for all $i$, and with relation (5) that $\alpha_{i}^{(2)}=0$ for all $i$.

By relation (2) $\left(\Gamma_{s}\right.$ commutes with $\left.\Gamma_{x_{i}}\right)$ we have $\beta_{i}^{(1)}=0$, and relation (3) gives $\beta_{i}^{(2)}=0$. So $\Gamma$ must verify:

$$
\begin{array}{rrrrrr}
s \rightarrow \Gamma_{s} & = & s+\beta_{1} t & +\sum_{j=1}^{n} \gamma_{j}^{(s, 1)} x_{j}+\sum_{j=1}^{n} \gamma_{j}^{(s, 2)} \partial_{j} \\
t \rightarrow \Gamma_{t} & = & \beta_{2} t & \\
x_{i} \rightarrow \Gamma_{x_{i}} & = & & \sum_{j=1}^{n} \gamma_{i, j}^{(1,1)} x_{j}+\sum_{j=1}^{n} \gamma_{i, j}^{(1,2)} \partial_{j} \\
\partial_{i} \rightarrow \Gamma_{\partial_{i}}= & & \sum_{j=1}^{n} \gamma_{i, j}^{(2,1)} x_{j}+\sum_{j=1}^{n} \gamma_{i, j}^{(2,2)} \partial_{j}
\end{array}
$$

Due to relations from (6) to (9) (between $\Gamma_{x_{i}}$ and $\Gamma_{\partial_{j}}$ ) we have that the submatrix

$$
\left(\begin{array}{ll}
\gamma_{i, j}^{(1,1)} & \gamma_{i, j}^{(1,2)} \\
\gamma_{i, j}^{(2,1)} & \gamma_{i, j}^{(2,2)}
\end{array}\right)
$$

verifies the relations of Lemma 4 , and in addition, from the relations with $\Gamma_{s}$ it verifies

$$
\sum \gamma_{i}^{(s, 1)} \gamma_{i, i}^{(1,2)}=\sum \gamma_{i}^{(s, 2)} \gamma_{i, i}^{(1,1)} \sum \gamma_{i}^{(s, 1)} \gamma_{i, i}^{(2,2)}=\sum \gamma_{i}^{(s, 2)} \gamma_{i, i}^{(2,1)}
$$

Anyway if we take for example $t x_{1}$, the requirements for $\Gamma$ produce

$$
\Gamma_{t x_{1}}=\beta_{2} t \Gamma_{x_{1}} \neq v^{2}+\widetilde{\Gamma_{t x_{1}}} .
$$

Thus we can not repeat the second step of the process in our PBW algebra in the same way that appears in [Gr1].

Problem 1. Find a general bound for the solutions of a general linear system over any PBW algebra or, at least, give such a bound for $R$.

We will not treat this general problem: with the aim of obtaining a bound for the complexity of the annihilating ideal of $f^{s}$, we will consider only the particular case of one equation of the type that would produce the definition of the ideal $I$ in section 2.1 or $I^{\prime}$ in section 2.2. In both cases we are interested in the complexity of computing their Gröbner bases (in different rings), and we do it considering the equivalent problem of computing the syzygies of the generators of our respective ideals. 
Remark 1. In the algorithm of [OT1] the calculations are computed in a Weyl algebra of $2 n+4 p$ variables in all, or more precisely in a commutative polynomial ring with $n+3 p,(x, u, v, t)$ commutative variables extended with $n+p,\left(\partial_{x}, \partial_{t}\right)$ "differential" variables. Let us denote by $A$ this algebra. The complexity of computing the annihilating ideal of $f^{s}$ is bounded by the complexity of computing a Gröbner basis in $A$.

Recall that the complexity in the Weyl algebra is given by the following theorem:

Theorem 1 (Th. 6,[Gr1]). Given a solvable system in the Weyl algebra $D_{n}$ :

$$
\sum_{1 \leq l \leq s} u_{k, l} V_{l}=w_{k}, \quad 1 \leq k \leq m
$$

with $\operatorname{deg}\left(u_{k, l}\right), \operatorname{deg}\left(w_{k}\right) \leq d$. There exists a solution with $\operatorname{deg}\left(V_{l}\right)<(m d)^{2^{O(n)}}$

As we said before in the Briançon-Maisonobe ring $R$ we can not construct a similar algorithm to bound the degree of a solution for a system in general. But in our very special case, our problem is equivalent to computing the solutions of the equation:

$\left(s_{1}+f_{1} t_{1}\right) V_{1}+\ldots+\left(s_{p}+f_{p} t_{p}\right) V_{p}+\left(\partial_{1}+\sum_{j} \frac{\partial f_{j}}{\partial x_{1}} t_{j}\right) V_{p+1}+\ldots+\left(\partial_{n}+\sum_{j} \frac{\partial f_{j}}{\partial x_{n}} t_{j}\right) V_{p+n}=0$

To simplify notation we write the precedent equation as

$$
\sum_{l} Q_{l} V_{l}=0
$$

Theorem 2. Given $f=\left(f_{1}, \ldots, f_{p}\right)$, the complexity of the computation of the annhilating ideal of $f^{s}$ in the Briançon-Maisonobe algebra $R=D_{n}\left[s_{1}, \ldots, s_{p}, t_{1}, \ldots, t_{p}\right]$ is bounded by the complexity of the computation of the syzygies of the elements $\partial_{i}+\sum_{j} \frac{\partial f_{j}}{\partial x_{i}} t_{j}$ in the Weyl algebra $D_{n}\left[t_{1}, \ldots, t_{p}\right]$.

Proof. We follow the notations of [Gr1] in this proof. We first compute $h_{1}^{(l)}, h^{(l)}$ for $2 \leq l \leq n+p$ such that:

$$
\begin{array}{rll}
\left(s_{1}+f_{1} t_{1}\right) h_{1}^{(2)} & +\left(s_{2}+f_{2} t_{2}\right) h^{(2)} & =0 \\
& \vdots & \\
\left(s_{1}+f_{1} t_{1}\right) h_{1}^{(p)} & +\left(s_{p}+f_{p} t_{p}\right) h^{(p)} & =0 \\
\left(s_{1}+f_{1} t_{1}\right) h_{1}^{(p+1)} & +\left(\partial_{1}+\sum_{j} \frac{\partial f_{j}}{\partial x_{1}} t_{j}\right) h^{(p+1)} & =0 \\
& \vdots & \\
\left(s_{1}+f_{1} t_{1}\right) h_{1}^{(p+n)} & +\left(\partial_{n}+\sum_{j} \frac{\partial f_{j}}{\partial x_{n}} t_{j}\right) h^{(p+n)} & =0 .
\end{array}
$$

The aim of these $h^{(l)}$ is to reduce any solution $V=\left(V_{1}, \ldots, V_{p+n}\right)$ of equation (2) to another one without $s_{1}$ from which you can recover $V$. The process will be repeated for $s_{2}, \ldots, s_{p}$.

It is easy to see that

$$
\begin{gathered}
{\left[s_{i}+f_{i} t_{i}, s_{j}+f_{j} t_{j}\right]=0} \\
{\left[s_{i}+f_{i} t_{i}, \partial_{j}+\sum_{l} \frac{\partial f_{l}}{\partial x_{j}} t_{l}\right]=s_{i}\left(\sum_{l} \frac{\partial f_{l}}{\partial x_{j}} t_{l}\right)+f_{i} t_{i} \partial_{j}-\partial_{j} f_{i} t_{i}-\left(\sum_{l} \frac{\partial f_{l}}{\partial x_{j}} t_{l}\right) s_{i}=}
\end{gathered}
$$




$$
=t_{i} s_{i} \frac{\partial f_{i}}{\partial x_{j}}+t_{i} \frac{\partial f_{i}}{\partial x_{j}}+\sum_{l \neq i} t_{l} s_{i} \frac{\partial f_{l}}{\partial x_{j}}+t_{i} f_{i} \partial_{j}-t_{i} f_{i} \partial_{j}-t_{i} \frac{\partial f_{i}}{\partial x_{j}}-\sum_{l} \frac{\partial f_{l}}{\partial x_{j}} t_{l} s_{i}=0 .
$$

Let us define $h^{(l)}=s_{1}+f_{1} t_{1}$ for all $l \geq 2$. We make the division of the $V_{l}$ of equation $(2), l \geq 2$ by $h^{(l)}$ with respect to a lexicographical ordering with $s_{1}$ greater than any other variable. We obtain a remainder $\bar{V}_{l}$ such that $\operatorname{deg}_{s_{1}}\left(\bar{V}_{l}\right)<\operatorname{deg}_{s_{1}}\left(h^{(l)}\right)=1$, so it has no $s_{1}$. So $V_{l}=h^{(l)} \overline{\bar{V}}_{l}+\bar{V}_{l}$, and adding the relation $Q_{1} h_{1}^{(l)}+Q_{l} h^{(l)}=0$ multiplied by $-\overline{\bar{V}}_{l}$ to equation (2), we obtain:

$$
Q_{1} \bar{V}_{1}+Q_{2} \bar{V}_{2}+\cdots+Q_{n+p} \bar{V}_{n+p}=0
$$

with $Q_{i}, \bar{V}_{i}$ without $s_{1}$ for $i \geq 2$, so $\bar{V}_{1}=0$, where $\bar{V}_{1}=V_{1}-h_{1}^{(2)} \overline{\bar{V}}_{2}-\cdots-h_{1}^{(n+p)} \overline{\bar{V}}_{n+p}$, and we have the new equation:

$$
Q_{2} \bar{V}_{2}+\cdots+Q_{n+p} \bar{V}_{n+p}=0
$$

in a Briançon-Maisonobe algebra $\mathbf{C}\left[s_{2}, \ldots, s_{p}, t_{1}, \ldots, t_{p}, x, \partial\right]$.

Repeating the process for $Q_{2}, \ldots, Q_{p}$, we reduce our problem to solving:

$$
\left(\partial_{1}+\sum_{j} \frac{\partial f_{j}}{\partial x_{1}} t_{j}\right) V_{p+1}+\ldots+\left(\partial_{n}+\sum_{j} \frac{\partial f_{j}}{\partial x_{n}} t_{j}\right) V_{p+n}=0
$$

in the Weyl algebra $D_{n}\left[t_{1}, \ldots, t_{p}\right]$.

As a consequence of 2 , the bound for the complexity of computing the annihilating ideal of $f^{s}$ in $R$ is bounded by the complexity of computing a Gröbner basis in a Weyl algebra with $3 p$ variables less that the one required by the method of [OT1]. Although the complexity of computing these objects in any case is known to be double exponential (with respect to the number of variables and the total degree of the generators of the ideal) by Theorem 1, it is clear that the reduction of $3 p$ variables of [BM1] is a significant advantage in practice as it is shown in the examples (see section 3). The theoretical superiority of the method of [BM1] is an open problem.

Problem 2. Is the bound proposed in this work is reached a la Mayr-Meyer ([MM1])? (that is to say, find an example of annihilating ideal with this worst complexity).

\section{REFERENCES}

[Ba1] Bahloul, R. Algorithm for computing Bernstein-Sato ideals associated with a polynomial mapping. Journal of Symbolic Computation 32, 643-662, 2001.

[Be1] Bernstein, I.N. The analytic continuation of generalized functions with respect to a parameter. Funct. Anal. 6 273-285, 1972.

[Bj1] Bjork, J.E. Dimensions over Algebras of Differential Operators. Preprint, 1973.

[BM1] Briançon, J. and Maisonobe, Ph. Remarques sur l'idéal de Bernstein associé à des polynômes. PUMA, 650 (preprint). 2002.

[BS1] Budur, N. and Saito M. Multiplier ideals, $V$-filtration and spectrum. arXiv:math.AG/0305118, 2003

[BGV1] Bueso, J.L. Goméz-Torrecillas, J. and Verschoren, A. Algorithmic methods in noncommutative algebra. Applications to quantum groups. Mathematical modelling. Theory and applications 17, Kluwer Academic Publishers. 2004.

[GHU1] Gago-Vargas, J., Hartillo-Hermoso, M.I. and Ucha-Enríquez, J.M. On the computation of the annihilating ideal of a product of polynomials. In preparation.

[GLS1] G.-M. Greuel, V. Levandovskyy, and H. Schönemann. Singular::Plural 2.1. A Computer Algebra System for Noncommutative Polynomial Algebras. Centre for Computer Algebra, University of Kaiserslautern (2003). http://www.singular.uni-kl.de/plural. 
[Gr1] Grigoriev, D. Complexity of solving systems of linear equations over the rings of differential operators. In Effective methods in algebraic geometry (Castiglioncello, 1990), Progr. Math., 94. Birkhauser Boston, MA 195-202. 1991.

[H1] Hamm, H.A. Remarks on asymptotic integrals, the polynomial of I.N. Bernstein and the Picard-Lefschetz monodromy. In Several complex variables (Proc. Sympos. Pure Math. Vol XXX, Part 1, Williams Coll., Williamstown, Mass. 1975), 31-35. Amer. Math. Soc., Providence, R.I. 1977.

[K1] Kashiwara, M. B-functions and holonomic systems. Rationality of roots of $B$-functions. Invent. Math. 38 (1) 33-53. 1976.

[L1] Lichtin, B. Generalized Dirichlet series and b-functions. Compositio Math 65 81-120. 1988.

[Mal1] Malgrange, B. Le polynôme de Bernstein d'une singularité isolée. In Fourier integral operators and partial differential equations (Colloq. Internat., Univ. Nice, Nice, 1974). Lecture Notes in Math. 459 Springer-Verlag, New York 98-119. 1975.

[MM1] Mayr, E.W. and Meyer, A.R. The complexity of the word problems for commutative semigroups and polynomial ideals. Adv. in Math. 46(3), 305-329. 1982.

[N1] M. Noro, T. Shimoyama and Takeshima, T. A Computer Algebra System Risa/Asir (2000). Available at ftp://archives.cs.ehime-u.ac.jp/pub/asir2000/.

[O1] Oaku, T. An algorithm of computing b-functions. Duke Math. J. 87 115-132 1997.

[OT1] Oaku, T. Takayama, N. An algorithm for de Rham cohomology groups of the complement of an affine variety via D-module computation. Journal of Pure and Applied Algebra 139 201-233 1999.

[Sa1] Sabbah, C. Proximité évanescente I. La structure polaire d'un D-Module. Apendice en collaboration avec F.J. Castro Jiménez. Compositio Math. 62, 283-328. 1987.

[Sa2] Sabbah, C. Proximité évanescente II. Equations fonctionelles pour plusieurs fonctions analytiques. Compositio Math. 64, 213-241. 1987.

[Se1] Seidenberg, A. Constructions in algebra. Trans. Amer. Math. Soc. 197 273-313. 1974.

[St1] Stafford, J. T. Module structure of Weyl algebras. J. London Math. Soc. (2) 18, no. 3, 429-442. 1978.

Depto. de Álgebra, Universidad de Sevilla. Apdo. 1160, E-41080 Sevilla (Spain)

E-mail address: gago@algebra.us.es

Depto. de Matemáticas, Universidad de Cádiz. Apdo. 40, E-11510 Puerto Real (Spain)

E-mail address: isabel.hartillo@uca.es

Depto. de Álgebra, Universidad de Sevilla. Apdo. 1160, E-41080 Sevilla (Spain)

E-mail address: ucha@us.es 